\title{
Nuclear localization of glutamate-cysteine ligase is associated with proliferation in head and neck squamous cell carcinoma
}

\author{
DIDIER DEQUANTER ${ }^{1}$, MAUREEN VAN DE VELDE ${ }^{2}$, ISABELLE BAR ${ }^{3}$, \\ VINCENT NUYENS ${ }^{4}$, ALEXANDRE ROUSSEAU $^{4}$, NATHALIE NAGY $^{5}$, LUC VANHAMME $^{6}$, \\ MICHEL VANHAEVERBEEK ${ }^{7}$, DANY BROHÉE ${ }^{8}$, PAUL DELRÉE ${ }^{3}$, KARIM ZOUAOUI BOUDJELTIA ${ }^{4}$, \\ PHILIPPE LOTHAIRE ${ }^{1^{*}}$ and PIERRICK UZUREAU ${ }^{4 *}$
}

\begin{abstract}
${ }^{1}$ Department of Surgery, University Hospital Center of Charleroi, André Vésale Hospital, Université Libre de Bruxelles, Montigny-le-Tilleul B-6110; ${ }^{2}$ Interdisciplinary Cluster for Applied Genoproteomics, University of Liège, Liège B-4000;

${ }^{3}$ Department of Pathology, Institute of Pathology and Genetics, Gosselies B-6041;

${ }^{4}$ Laboratory of Experimental Medicine (ULB222), André Vésale Hospital, Université Libre de Bruxelles, Montigny-le-Tilleul B-6110; ${ }^{5}$ Department of Pathological Anatomy, University Hospital Center in Charleroi,

André Vésale Hospital, Université Libre de Bruxelles, Montigny-le-Tilleul B-6110; ${ }^{6}$ Laboratory of Molecular Parasitology, Institute of Molecular Biology and Medicine, Université Libre de Bruxelles, Charleroi B-6041; Departments of ${ }^{7}$ Internal Medicine and ${ }^{8}$ Oncology, University Hospital Center in Charleroi, André Vésale Hospital, Université Libre de Bruxelles, Montigny-le-Tilleul B-6110, Belgium
\end{abstract}

Received March 1, 2015; Accepted February 12, 2016

DOI: $10.3892 / \mathrm{ol} .2016 .4458$

\begin{abstract}
Glutathione (GSH) is the keystone of the cellular response toward oxidative stress. Elevated GSH content correlates with increased resistance to chemotherapy and radiotherapy of head and neck $(\mathrm{HN})$ tumors. The purpose of the present cross-sectional study was to evaluate whether the expression of glutamate-cysteine ligase (GCL) accounts for the increased GSH availability observed in HN squamous cell carcinoma (SCC). For that purpose, the messenger (m)RNA levels of the modifier (M) and catalytic (C) subunits of GCL and its putative regulators (namely, nuclear factor erythroid 2-related factor 2, heme oxygenase-1 and nuclear factor of kappa light polypeptide gene enhancer in B-cells inhibitor, alpha) were monitored in 35 surgical resections of untreated HNSCC. The localization of GCLM was evaluated using in situ hybridization and immunohistochemistry. GCLM expression was significantly increased in tumor samples, compared with normal mucosa, both at the mRNA and protein level $(\mathrm{P}=0.029)$, but the pathway of GCLM activation remains to be elucidated. Protein expression of GCLM was detected
\end{abstract}

Correspondence to: Dr Pierrick Uzureau, Laboratory of Experimental Medicine (ULB222), André Vésale Hospital, Unit 222, Université Libre de Bruxelles, Route de Gozée 706, Montigny-le-Tilleul B-6110, Belgium

E-mail: puzureau@ulb.ac.be

${ }^{*}$ Contributed equally

Key words: head and neck squamous cell carcinoma, oxidative stress, glutathione, glutamate-cysteine ligase, Ki-67 antigen in the cytoplasm and nucleus. GCLM and the proliferation marker Ki-67 displayed a similar distribution, being both mainly expressed at the periphery of tumor lobules. The present study reported increased expression of GCL and the rate-limiting enzyme of GSH synthesis, within HNSCC. The nuclear localization of GCLM and the concomitant expression of $\mathrm{Ki}-67$ suggested that the localization of GSH synthesis contributes to the protection against oxidative stress within hotspots of cell proliferation.

\section{Introduction}

Cancer of the head and neck (HN), primarily squamous cell carcinoma (SCC) of the oral cavity, and cancer of the pharynx and larynx account for $6 \%$ of all malignancies (1). In the case of pharyngolaryngeal cancer, radiotherapy and chemotherapy are currently accepted as an alternative approach to surgery for patients with advanced HNSCC, since it enables organ preservation without compromising patient survival (2). However, the response to chemotherapy and radiotherapy is heterogeneous, and a large proportion of patients relapse, either locally or at distant sites, resulting in a 5-year survival rate of $50 \%(1,2)$. Chemotherapy and radiotherapy share common downstream effectors, namely reactive oxygen species (ROS) (3). Although ROS toxicity for tumor cells is well established, the activation of the oxidative stress pathway also favors the development and spreading of certain tumors; thus, oxidative stress exhibits a Janus-head effect in terms of cancer progression $(3,4)$.

In normal cells, glutathione (GSH) is one of the main ROS scavenging molecules, and is important in the cellular response to oxidation (4). GSH is synthesized following a two-step reaction, by coupling three amino acids, namely, cysteine, glutamine and glycine (5). Under normal conditions, 
the levels of GSH depend on the efficiency of the first step of the synthesis reaction, which is performed by the enzyme glutamate-cysteine ligase (GCL) (5). GCL is composed of two subunits, namely the catalytic (C) subunit and the modulator (M) subunit (5). GCL activity only requires the GCLC subunit, but it is strongly induced by the GCLM subunit (6). These two GCL subunits exhibit different pattern of expression within tissues, which suggests an independent control of their expression (7). Notably, although only the expression of GCLC is altered upon stimulation with hormones or drugs, the expression of both subunits is induced following exposure of cells to oxidative stress $(8,9)$. The promoters of GCLC and GCLM harbor binding sites for three transcription factors that have been associated with the induction of the oxidative stress response machinery (10-12). These transcription factors are nuclear factor erythroid 2-related factor 2 (NRF2), nuclear factor (NF) $-\kappa B$ and activator protein-1 (AP-1) (13). Previous functional assays have reported the regulation of the transcription of the GCL subunits genes by the transcription factors NRF2 and AP-1 and by members of the NF- $\kappa$ B signaling pathway $(14,15)$. The NRF2 signaling pathway is a prominent regulator of the cellular response to oxidative stress (16). In the absence of oxidative stress, Kelch-like erythroid cell-derived protein with cap'n'collar homology-associated protein 1 (KEAP1) recruits NRF2, and the KEAP1/NRF2 complex is then targeted to the proteasome (16). Oxidation of cysteine residues in KEAP1 prevents the formation of the complex (13). Upon stabilization of the complex, NRF2 is translocated to the nucleus, where it triggers the transcription of the genes of phase II detoxifying enzymes, including the aforementioned GCL subunits and heme oxygenase-1 (HO-1) (14,17).

Considering the role of GSH in ROS detoxification, the present and other authors have previously attempted the quantification of GSH within tumors, compared with normal tissues $(18,19)$. In agreement with previous studies reporting the accumulation of GSH within various tumors, the present authors have recently reported a higher ratio of reduced vs. oxidized GSH in HN tumors, compared with the adjacent mucosa (19). The aim of the present study was to evaluate the expression of GCL, the rate-limiting enzyme of GSH synthesis, in carcinoma tissues, compared with adjacent mucosa. For that purpose, the messenger (m)RNA and protein expression levels of the two GCL subunits and the mRNA levels of their regulators were measured in biopsies of HN tumors that had not been treated with radiotherapy or chemotherapy, in order to avoid any potential interference with oxidative stress that may have been induced by these therapies.

\section{Materials and methods}

Ethics statement. The present study was approved by the Ethics Committee of André Vésale Hospital (Intermunicipal Public Health of the Charleroi registration number OM008; Montigny-le-Tilleul, Belgium) under Compliance Certification Board number B32520107991 and B325201111821.

Clinical data. Biopsy samples from carcinoma tissues and adjacent normal tissues were collected from patients who had undergone surgical resection of HNSCC at the André Vésale Hospital (Montigny-le-Tilleul, Belgium) between 2011 and
2013 (Table I). Only patients who had not been previously subjected to chemotherapy or radiotherapy were included in the study. Cancer stages of the patients ranged from stage II to IV (Table I), according to the tumor-node-metastasis classification of malignant tumors (20). Patient's tumors were localized in the oral cavity, hypopharynx and larynx, and ranged from poorly to well differentiated (Table I).

Sample collection. Fresh samples and formalin-fixed, paraffin-embedded (FFPE) tissue sections of tumor and adjacent normal tissues were collected from surgical resections of HNSCC.

Reverse transcription-quantitative polymerase chain reaction (RT-qPCR). Immediately following resection, samples for RNA extraction were collected, frozen in liquid nitrogen and stored at $-80^{\circ} \mathrm{C}$. Tissue samples were grinded with a mortar in a liquid nitrogen bath (Bel-Art Products, Wayne, NJ, USA). RNA extraction was performed using RNeasy Mini kit (Qiagen, Inc., Valencia, CA, USA), according to the manufacturer's protocol, and including DNAse treatment (Qiagen, Inc.).

RT-qPCR was performed using total RNA. Complementary DNA was synthesized with Transcriptor Reverse Transcriptase (Roche Diagnostics, Indianapolis, IN, USA) using oligo(dT) primers (Qiagen, Inc.), according to the manufacturer's protocol. RT-qPCR was conducted with the primer sets presented in Table II (Sigma-Aldrich, St. Louis, MO, USA), using SYBR Green I Master (Roche Diagnostics), according to the manufacturer's protocol, in a LightCycler ${ }^{\circledR}$ 480 Instrument II (Roche Diagnostics). The cycle conditions were $95^{\circ} \mathrm{C}$ for $5 \mathrm{~min}$, followed by 50 cycles of $95^{\circ} \mathrm{C}$ for $15 \mathrm{sec}$, $60^{\circ} \mathrm{C}$ for $30 \mathrm{sec}$ and $72^{\circ} \mathrm{C}$ for $30 \mathrm{sec}$. Relative expression (RE) of GCLM, GCLC, NRF2, HO-1 and nuclear factor of kappa light polypeptide gene enhancer in B-cells inhibitor, alpha (NFKBIA) was calculated using succinate dehydrogenase complex flavoprotein subunit $\mathrm{A}$ and ribosomal protein L27 as reference genes, according to the following formula: $\mathrm{RE}=2^{\mathrm{Cq} \text { (reference)-Cq (target) }}$ (21). Analyses of GCLC, NRF2, HO-1 and NFKBIA expression were restricted to 21, 24, 24 and 22 patients, respectively, since certain tissues samples collected for RNA extraction were not sutible for qPCR analysis due to RNA degradation. A no template control and no reverse transcriptase control were performed to exclude extraneous nucleic acid contamination and genomic DNA contamination, respectively.

Immunohistochemistry (IHC). IHC was performed on 5- $\mu \mathrm{m}$ paraffin-embedded, $10 \%$ formalin-fixed tissue sections from 6 patients (Table I). Tissue sections were deparaffinized during heat-induced antigen retrieval, which was conducted in EnVision ${ }^{\mathrm{TM}}$ Flex Target Retrieval Solution High $\mathrm{pH}$ (catalog no., K8004; Dako, Glostrup, Denmark) for $10 \mathrm{~min}$ at $97^{\circ} \mathrm{C}$, using the PT Link apparatus (Dako), followed by a 20-min cool down period and wash in Tris-buffered saline (Sigma-Aldrich). All subsequent steps were performed using the EnVision ${ }^{\mathrm{TM}}$ FLEX/HRP kit (Dako) according to the manufacturer's protocol, which includes the diaminobenzidine (DAB) substrate. Polyclonal rabbit anti-GCLM (dilution, 1:40; catalog no., HPA023696; Sigma-Aldrich) was incubated overnight at $4^{\circ} \mathrm{C}$ with the tissue slides for GCLM detection. 
Table I. Patient's clinical data.

\begin{tabular}{|c|c|c|c|c|c|}
\hline Gender & Age, years & Surgery date, month/year & TNM stage & Localization & SSC grade $^{b}$ \\
\hline M & 48 & $02 / 2013$ & T4N2 & Larynx & III \\
\hline M & 49 & $03 / 2013$ & T2NO & Mobile tongue & I \\
\hline M & 72 & $06 / 2013$ & T4N1 & Larynx & II \\
\hline M & 55 & 07/2012 & T4N0 & Larynx & I \\
\hline M & 57 & $07 / 2012$ & T2N0 & Mobile tongue & I \\
\hline M & 62 & $07 / 2012$ & $\mathrm{~T} 2 \mathrm{~N} 2$ & Oropharynx & I \\
\hline M & 58 & $08 / 2012$ & T4N0 & Larynx & I \\
\hline M & 57 & 09/2011 & T4N2 & Mobile tongue & I \\
\hline M & 85 & 09/2011 & T4NO & Larynx & I \\
\hline $\mathrm{F}$ & 84 & $10 / 2011$ & T2N0 & Oropharynx & III \\
\hline M & 66 & $11 / 2011$ & $\mathrm{~T} 4 \mathrm{~N} 2$ & Hypopharynx & III \\
\hline M & 75 & $10 / 2012$ & T4NO & Mobile tongue & $\mathrm{I}$ \\
\hline $\mathrm{F}$ & 73 & $10 / 2012$ & T4N0 & Mobile tongue & II \\
\hline M & 54 & $11 / 2012$ & $\mathrm{~T} 4 \mathrm{~N} 2$ & Larynx & I \\
\hline $\mathrm{F}$ & 68 & $11 / 2012$ & $\mathrm{~T} 2 \mathrm{~N} 1$ & Mobile tongue & I \\
\hline $\mathrm{F}$ & 63 & $12 / 2012$ & T2NO & Oropharynx & I \\
\hline M & 78 & $01 / 2013$ & T4N0 & Oropharynx & II \\
\hline M & 50 & $01 / 2013$ & T4N1 & Floor of the mouth & III \\
\hline M & 62 & $02 / 2013$ & T2NO & Mobile tongue & I \\
\hline M & 58 & $05 / 2013$ & T2N0 & Oropharynx & I \\
\hline M & 72 & $06 / 2013$ & T4N1 & Larynx & II \\
\hline M & 54 & $04 / 2013$ & T4N2 & Oropharynx & III \\
\hline M & 58 & $04 / 2013$ & $\mathrm{~T} 4 \mathrm{~N} 2$ & Larynx & $\mathrm{I}$ \\
\hline M & 59 & 09/2013 & T4N0 & Oropharynx & I \\
\hline M & 54 & $10 / 2013$ & T3N2 & Floor of the mouth & I \\
\hline M & 67 & $11 / 2013$ & T4NO & Larynx & I \\
\hline M & 51 & $11 / 2013$ & $\mathrm{~T} 4 \mathrm{~N} 2$ & Hypopharynx & III \\
\hline M & 50 & $12 / 2013$ & T4N0 & Larynx & I \\
\hline M & 63 & $03 / 2013$ & $\mathrm{~T} 4 \mathrm{~N} 2$ & Larynx & I \\
\hline $\mathrm{F}^{\mathrm{a}}$ & 75 & $07 / 2013$ & $\mathrm{~T} 4 \mathrm{~N} 2$ & Larynx & II \\
\hline $\mathrm{F}^{\mathrm{a}}$ & 89 & 08/2013 & T2NO & Oropharynx & I \\
\hline $\mathrm{M}^{\mathrm{a}}$ & 63 & $05 / 2013$ & T4N0 & Hypopharynx & II \\
\hline $\mathrm{M}^{\mathrm{a}}$ & 61 & 07/2013 & T4N1 & Larynx & I \\
\hline $\mathrm{M}^{\mathrm{a}}$ & 59 & $09 / 2013$ & T4N1 & Larynx & I \\
\hline $\mathrm{F}^{\mathrm{a}}$ & 58 & $09 / 2013$ & T2N0 & Oropharynx & I \\
\hline
\end{tabular}

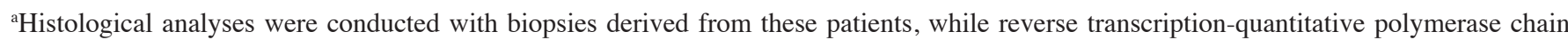
reaction analysis was performed with data derived from biopsies of all the patients listed in the table. 'SCC grade indicated: I, well; II, moderately; and III, poorly differentiated tumor. M, male; F, female; TNM, tumor-node-metastasis; SCC, squamous cell carcinoma.

Monoclonal mouse anti-MIB-1 antibody (undiluted; catalog no., IR626; Dako) was incubated for $30 \mathrm{~min}$ at room temperature with the tissue slides for Ki-67 detection. Normal and tumor tissues were identified by trained pathologists (University Hospital Center of Charleroi, Charleroi and Institute of Pathology and Genetics, Gosselies, Belgium). Quantification of the signal in the different cell types was performed using 50 images captured on a Zeiss Axioplan microscope, using the 40X objective (Carl Zeiss AG, Oberkochen, Germany). Signal intensity was normalized using the white balance function of Adobe Photoshop CS2 software (Adobe Systems, Inc., San Jose, CA, USA) and the contrast enhancer of ImageJ software (National Institutes of Health, Bethesda, MD, USA), set at $0.1 \%$ saturated pixels. DAB signals were extracted using Image J and IHC Profiler plugin (22). Relative intensity was calculated as the mean gray value of the regions of interest subtracted from the maximum intensity value. The intensity of the GCLM signals was measured from the border to the center of each lobule using ImageJ and its dedicated macro, which is available at https://b2share.eudat.eu/record/149. In total, 60 lobules were analyzed as described for the different cell types, except that the signal intensity was measured within concentric selected areas of $10-\mu \mathrm{m}$ width from the border to the center of the selected lobule. The same procedure was 
Table II. List of the primers used for reverse transcription-quantitative polymerase chain reaction analysis.

\begin{tabular}{|c|c|}
\hline Gene & Primer sequence \\
\hline \multicolumn{2}{|l|}{ SDHA } \\
\hline Forward & 5'-CCCGAGGTTTTCACTTCACTGT-3' \\
\hline Reverse & 5'-CCAGTTGTCCTCCTCCATGTTC-3' \\
\hline \multicolumn{2}{|l|}{ RPL27 } \\
\hline Forward & 5'-ATCGCCAAGAGATCAAAGATAA-3' \\
\hline Reverse & 5'-TCTGAAGACATCCTTATTGACG-3' \\
\hline \multicolumn{2}{|l|}{ NRF2 } \\
\hline Forward & 5'-GCAAGTTTGGGAGGAGCTATTATC-3' \\
\hline Reverse & 5'-AGTTTGGCTTCTGGACTTGGA-3' \\
\hline \multicolumn{2}{|l|}{ GCLM } \\
\hline Forward & 5'-GAAGAGAGCATCTGGAGAACTAATGA-3' \\
\hline Reverse & 5'-AGTTATGACACTGTCTTGCTTGTAGTCA-3' \\
\hline \multicolumn{2}{|l|}{ GCLC } \\
\hline Forward & 5'-TTCCTGCACATCTACCACGC-3' \\
\hline Reverse & 5'-TGTATTCCACCTCATCGCCC-3' \\
\hline \multicolumn{2}{|l|}{ HO-1 } \\
\hline Forward & 5'-GCACTCAGGCAGAGGGTGATA-3' \\
\hline Reverse & 5'-CTGGAGTGTGCCCAATGCTAT-3' \\
\hline \multicolumn{2}{|l|}{ NFKBIA } \\
\hline Forward & 5'-CAATGCTCAGGAGCCCTGTAA-3' \\
\hline Reverse & 5'-TCTGTTGACATCAGCCCCAC-3' \\
\hline
\end{tabular}

SDHA, succinate dehydrogenase complex flavoprotein subunit A; RPL27, ribosomal protein L27; NRF2, nuclear factor erythroid 2-related factor 2; GCLM, glutamate-cysteine ligase modulator subunit; GCLC, glutamate-cysteine ligase catalytic subunit; HO-1, heme oxygenase 1; NFKBIA, nuclear factor of kappa light polypeptide gene enhancer in B-cells inhibitor, alpha.

applied to the quantification of Ki-67-labeled nuclei within 90 lobules, except for the following modification: The background was subtracted from the DAB signal image, and the image was converted to a binary image using the Rényi's entropy threshold (23) prior to nuclei count with the particle analyzer function of ImageJ.

In situ hybridization(ISH). GCLM mRNA was detected in FFPE tissues using the ISH kit RNAscope ${ }^{\circledR} 2.0$ (Advanced Cell Diagnostics Inc., Hayward, CA, USA) and the Probe - Hs-GCLM, target, 1 (catalog no., 411581; Advanced Cell Diagnostics Inc.), according to the manufacturer's protocol.

Statistical analyses. Statistical analyses were performed using SigmaPlot 12 software (Systat Software, Inc., San Jose, CA, USA). RT-qPCR data were analyzed using the Wilcoxon signed-rank test. Data relative to IHC labeling in the different cell types were analyzed using Kruskal-Wallis one-way analysis of variance (ANOVA) on ranks, followed by Dunn's test as a post hoc procedure for pairwise comparison. Statistical analysis of GCLM distribution was restricted to 24 lobules that delivered data within $0-100 \mu \mathrm{m}$ from the lobule edge, while statistical analysis of Ki-67 distribution was restricted to 34 lobules. Data were analyzed using repeated measures ANOVA on ranks (Friedman's test), followed by Dunnett's post hoc test vs. control.

\section{Results}

GCL mRNA levels in tumors. The mRNA expression levels of GCLM and GCLC were evaluated in biopsy samples from carcinoma and adjacent tissues. The mRNA expression levels of GCLM but not those of GCLC were significantly increased in tumor samples, compared with normal mucosa $(\mathrm{P}=0.029$; Fig. $1 \mathrm{~A}$ and $\mathrm{B}$ ). The role of the NRF2 and NF- $\kappa \mathrm{B}$ signaling pathways in GCLM activation was investigated in HNSCC tumors (Fig. 1C). The activation of the NRF2 signaling pathway was monitored by measuring the mRNA levels of NRF2, which have been demonstrated to be relevant for the activation of $\mathrm{NRF} 2$ in vivo (16). As the regulation of the NRF2 and NF- $\kappa \mathrm{B}$ signaling pathways involves post-translational modifications, the expression levels of HO-1 and NFKBIA were used as a reporter of NRF2 and $\mathrm{NF}-\kappa \mathrm{B}$ activity, respectively, since the HO-1 gene is under direct control of the transcription factor NRF2, while the transcription of the NF- $\kappa$ B inhibitor NFKBIA has been demonstrated to be a useful marker of NF- $\kappa \mathrm{B}$ activation $(17,24)$ (Fig. 1C). The present results indicated that the mRNA levels of NRF2 or HO-1 were not upregulated in the tumor samples, compared with adjacent normal mucosa (Fig. 1D and E), suggesting that the activity of the NRF2 pathway was not altered in the tumors. Regarding the NF- $\kappa \mathrm{B}$ pathway, both tumors and adjacent mucosa presented similar mRNA levels of NFKBIA (Fig. 1F). 
A

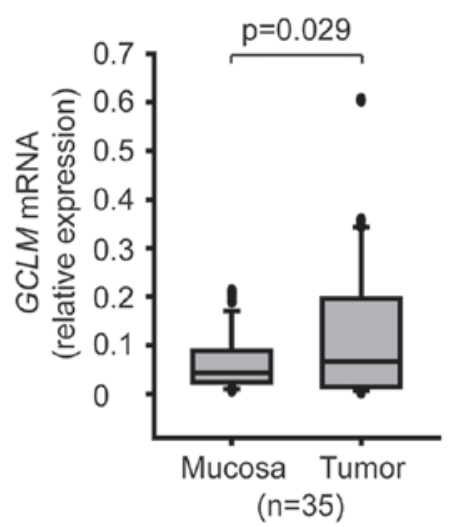

D

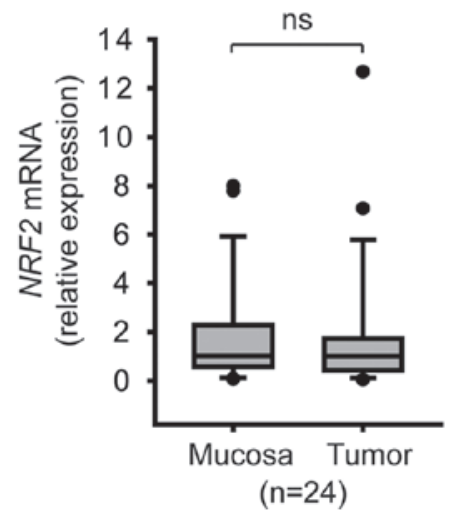

B

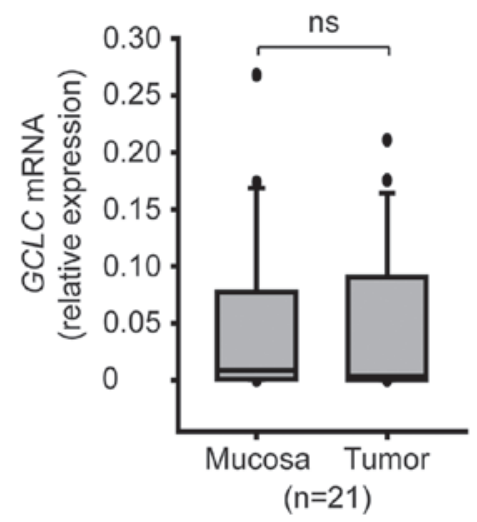

E

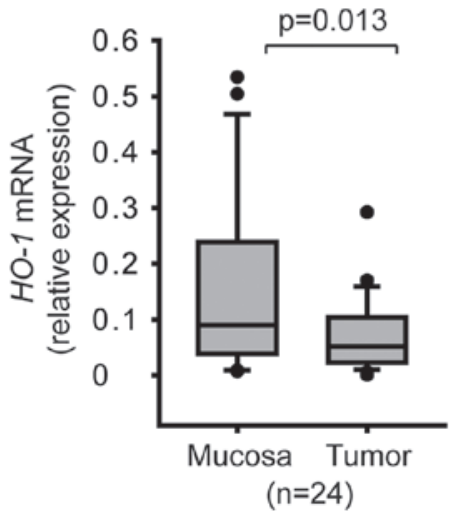

C

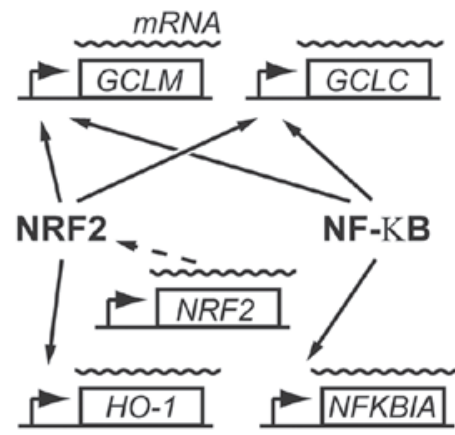

F

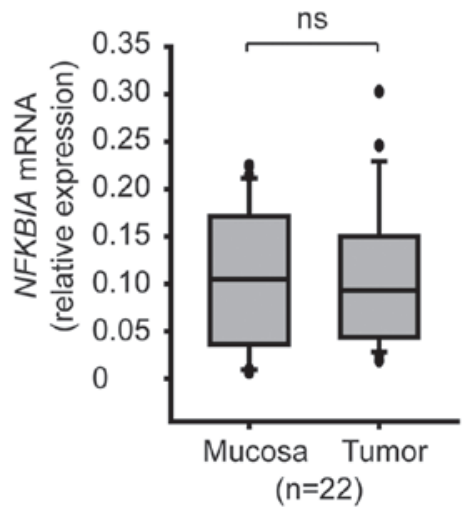

Figure 1. Expression of GCL subunits and regulators in tumor cells. Box plot graphs represent the relative mRNA expression levels of (A) GCLM, (B) GCLC, (D) NRF2, (E) heme oxygenase-1 and (F) nuclear factor of kappa light polypeptide gene enhancer in B-cells inhibitor, alpha in normal mucosa and tumor tissues. Total RNA was extracted from biopsy samples, and the corresponding mRNA levels were quantified by reverse transcription-quantitative polymerase chain reaction. The number of patients included in each analysis is shown in brackets. (C) Association between the genes of interest and the NRF2 and nuclear factor- $\kappa \mathrm{B}$ signaling pathways. SDHA, succinate dehydrogenase complex flavoprotein subunit A; RPL27, ribosomal protein L27; NRF2, nuclear factor erythroid 2-related factor 2; GCLM, glutamate-cysteine ligase modulator subunit; GCLC, glutamate-cysteine ligase catalytic subunit; HO-1, heme oxygenase-1; NFKBIA, nuclear factor of kappa light polypeptide gene enhancer in B-cells inhibitor, alpha; mRNA, messenger RNA; ns, not significant; NF- $\kappa$ B, nuclear factor- $\kappa \mathrm{B}$.

GCLM localization in tumors. The identification of cell types expressing GCLM mRNA within tumor samples was investigated at the mRNA and protein level. For that purpose, IHC of GCLM protein expression was performed on histological sections of tumors and adjacent mucosa. Within the normal epithelium, labeling was restricted to basal cells, whose cytoplasm and nucleus were both labeled, with the nuclei consistently presenting stronger labeling than the cytoplasms (Fig. 2A). In the case of pre-neoplastic lesions, dysplastic cells were labeled, with the nuclei exhibiting a stronger signal than the cytoplasms (Fig. 2B). GCLM labeling of the tumors was heterogeneous (Fig. 2C), but similarly to the findings in epithelial and dysplastic cells, GCLM was detected in the cytoplasm and nucleus of tumor cells (Fig. 2C and D). Systematic analysis of carcinoma lobules demonstrated that the mean GCLM labeling was comparable in normal basal cells, dysplasia and tumor lobules (Fig. 2E). The localization of GCLM protein correlated with the areas where the corresponding mRNA was detected, as indicated by the similar labeling patterns of the protein (Fig. 3A and B) and mRNA (Fig. 3C and D) expression in sequential histological sections. In both cases, while the borders of the tumor lobules were consistently labeled, the center exhibited a range of strong to very weak protein and mRNA signals (Fig. 2C). Systematic measurement of
GCLM labeling within the tumor lobules revealed a significant decrease in signal intensity from the periphery to regions located $\geq 50 \mu \mathrm{m}$ from the lobule edge (Fig. 4A and B). Based on previous studies reporting the peripheral localization of proliferative cells within HNSCC lobules $(25,26)$, the relative density of Ki-67-labeled nuclei within the HNSCC lobules was evaluated in the present study (Fig. 4C and D). The results revealed a consistent labeling of the corresponding regions with anti-GCLM and anti-Ki-67 antibodies, as illustrated by the correlation between the median values of both signals (Fig. 4E).

\section{Discussion}

Oxidative stress is the keystone of $\mathrm{HN}$ cancer therapy, which requires the administration of radiotherapy and/or chemotherapy for tumor treatment prior to or following surgical resection (2). Both strategies rely on the efficient induction of oxidative stress within the targeted cells, but inducing an associated oxidative stress response that will eventually salvage the cell $(3,4)$. Among the different salvage pathways, GSH is key in ROS detoxification, and has been demonstrated to be important in tumor resistance to the majority of chemotherapeutic drugs currently used against HN tumors $(27,28)$. By contrast, 
A

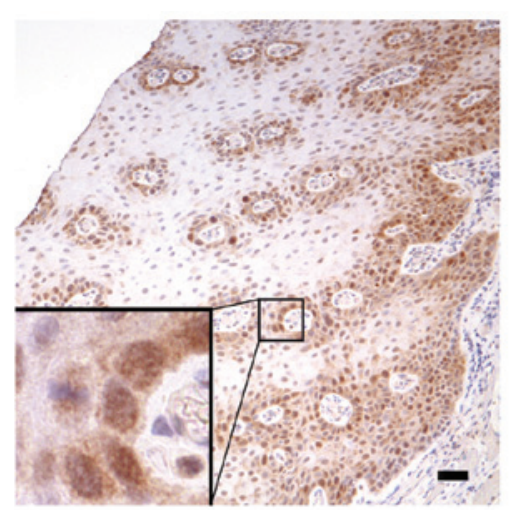

C

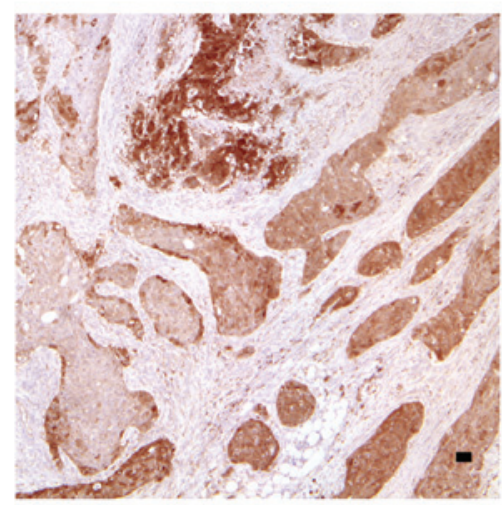

B

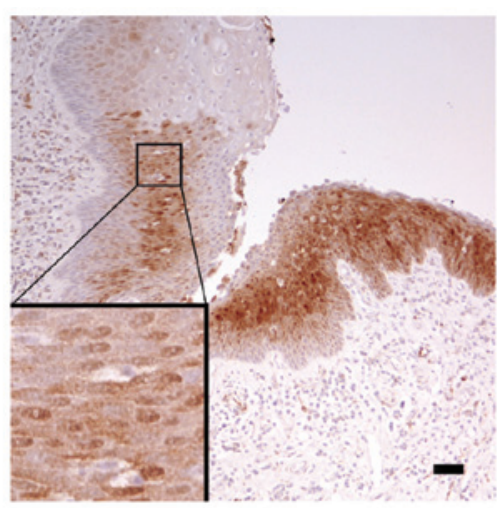

D

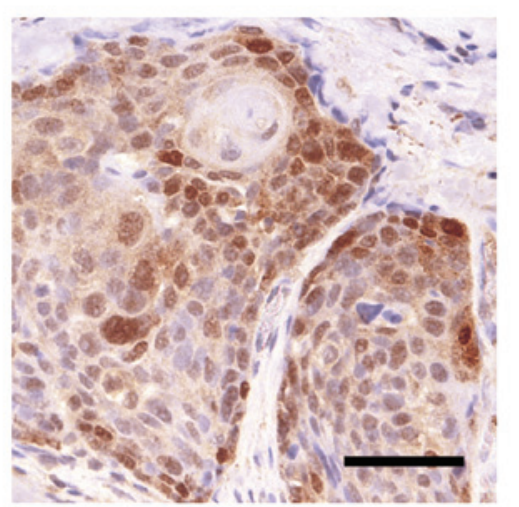

$\mathbf{E}$

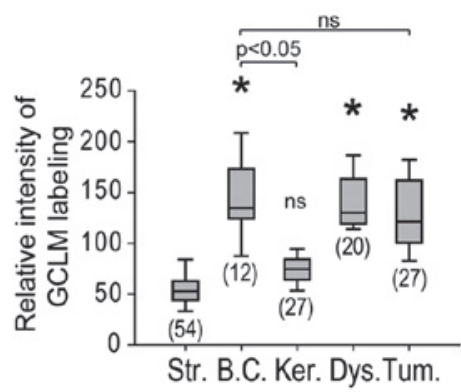

Figure 2. IHC staining of GCLM in (A) normal mucosa from resection margin (magnification, x60), (B) dysplasia within hemilarynx (magnification, x60) and (C and D) carcinoma (magnification C and D, x30 and x240, respectively). Boxes indicate enlarged regions. Scale bars correspond to $50 \mu \mathrm{m}$. (E) Relative intensity of IHC staining of GCLM in stroma, basal cells, keratinocytes, dysplastic cells and tumor. The number of regions analyzed for each tissue is shown in brackets. "P<0.05 vs. stroma. Str, stroma; BC, basal cells; Ker, keratinocytes; Dys, dysplastic cells; Tum, tumor; IHC, immunohistochemistry; GCLM, glutamate-cysteine ligase modulator subunit; ns, not significant.
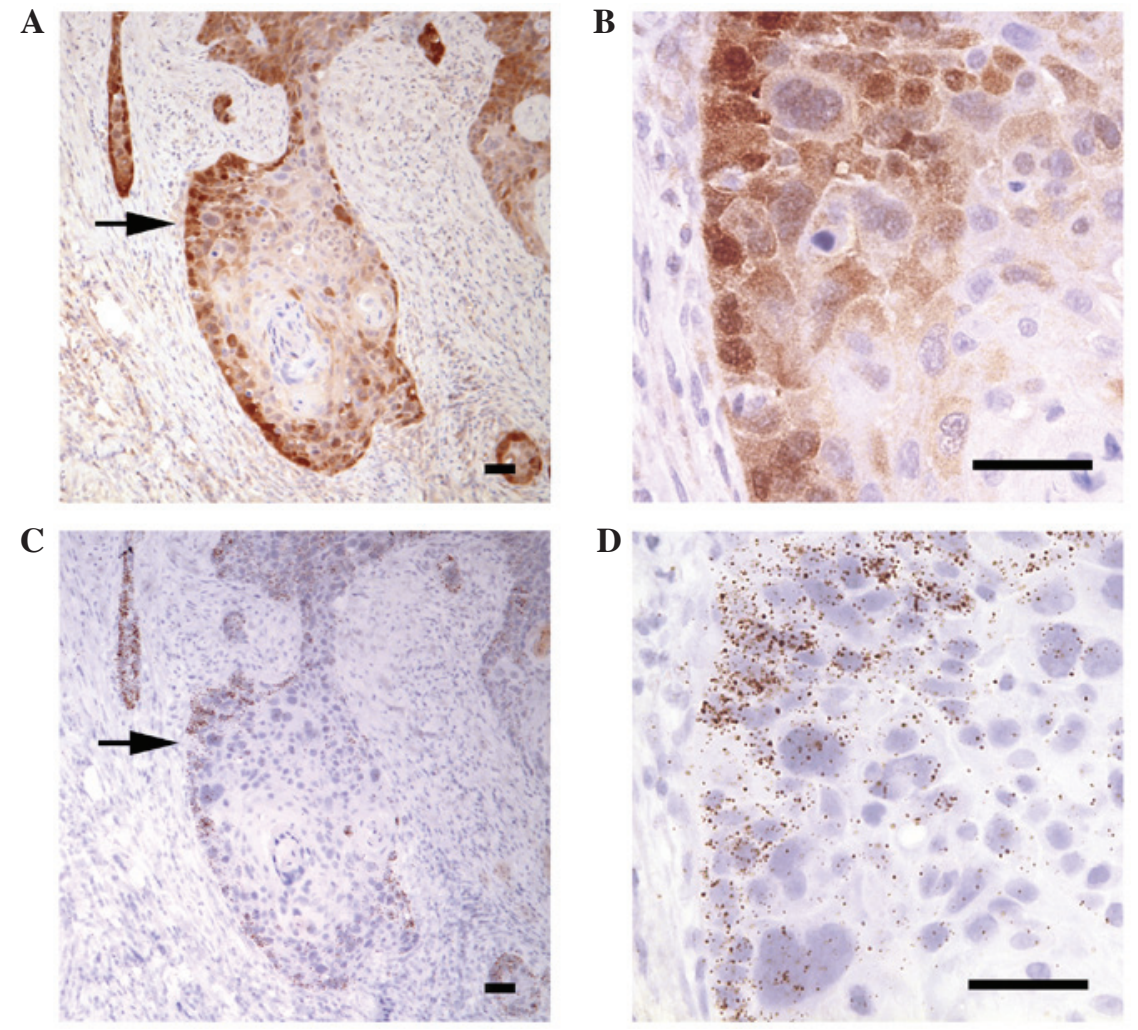

Figure 3. GCLM mRNA and protein expression in carcinoma. Protein and mRNA expression of GCLM were detected by (A and B) immunohistochemistry (magnification A and B, x60 and x240, respectively) and (C and D) in situ hybridization, respectively (magnification C and D, x60 and x240, respectively). The enlarged regions shown in panels B and D correspond to the areas indicated by a black arrow in panels A and C, respectively. The top and bottom panels correspond to the same region of a tissue section. Scale bars, $50 \mu \mathrm{m}$. mRNA, messenger RNA; GCLM, glutamate-cysteine ligase modulator subunit. 
A

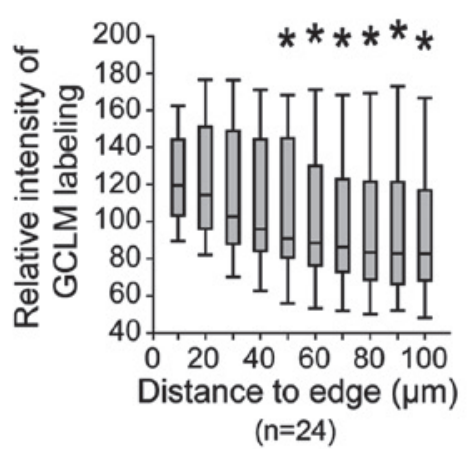

C

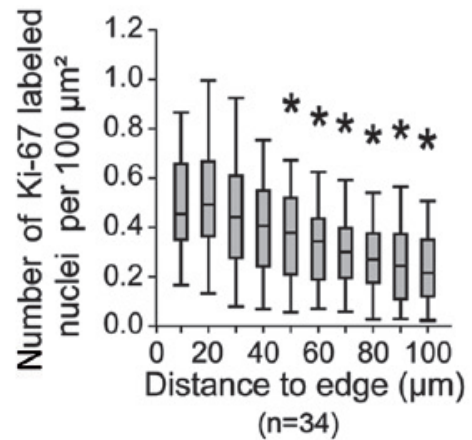

B

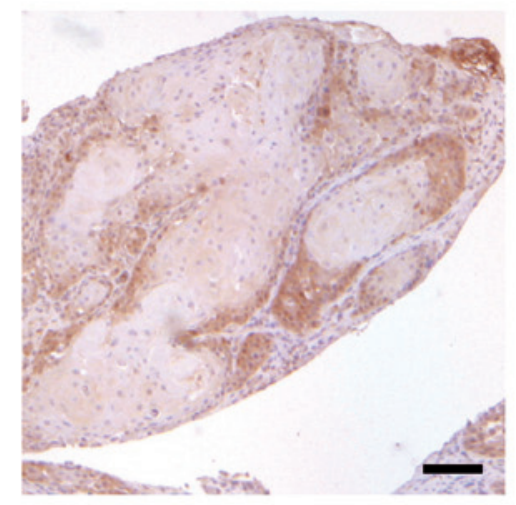

D

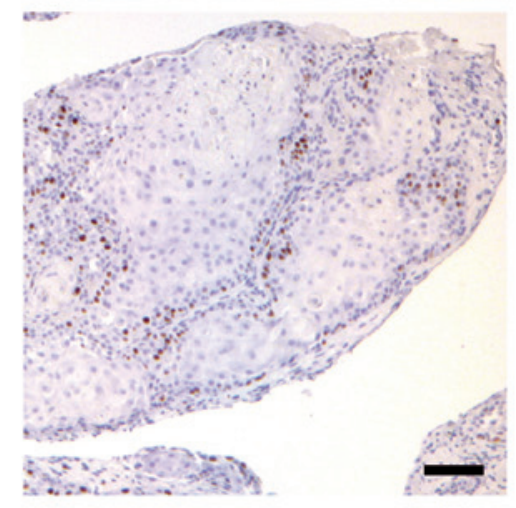

E

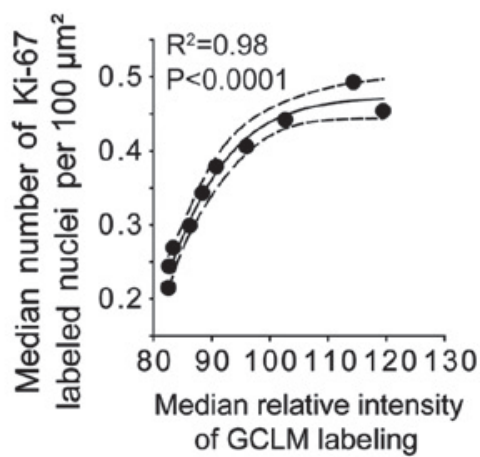

Figure 4. Peripheral expression of GCLM and Ki-67. (A) Relative quantification of GCLM labeling within concentric 10- $\mu \mathrm{m}$ wide slices from the periphery to the center of the tumor lobule. The number of lobules included in the analysis is shown in brackets. " $\mathrm{P}<0.05$ vs. $0-10 \mu \mathrm{m}$ range. (B) GCLM labeling within carcinoma tissue (magnification, x120). (C) Relative density of Ki-67-labeled nuclei (number of nuclei/100 $\mu \mathrm{m}^{2}$ ) within concentric $10-\mu \mathrm{m}$ wide slices from the periphery to the center of the tumor lobule. The number of lobules included in the analysis is shown in brackets. *P<0.05 vs. $0-10 \mu \mathrm{m}$ range. (D) Ki- 67 labeling within carcinoma tissue. The same region of a tissue section is presented in panels B and D. Scale bars, $50 \mu \mathrm{m}$; magnification, x120. (E) Association between GCLM and Ki-67 labeling. Dots correspond to the median values obtained from the 10- $\mu$ m wide slices of panels A and C. Sigmoidal Chapman regression curve and the corresponding $95 \%$ confidence interval are depicted by solid and dashed lines, respectively. GCLM, glutamate-cysteine ligase modulator subunit.

it is unclear whether the levels of GSH alter the outcome of radiotherapy. While certain studies have reported a correlation between the levels of GSH in blood and the efficiency of SCC treatment, the levels of GSH within the HN tumor itself do not appear to be associated with the degree of radiosensitivity exhibited by the tumor $(28,29)$. Thus, it may be hypothesized that cell fate may not only depend on the steady state levels of GSH, but also on the capability of the cell to induce the appropriate response against ROS damage. In order to evaluate this capability, the present study focused on the $\mathrm{C}$ and $\mathrm{M}$ subunits of GCL, the rate-limiting enzyme of GSH synthesis (5). While GCLC is sufficient to perform the first step of GSH synthesis, GCLM is an essential enhancer of GCLC activity, since it impairs the enzyme inhibition by GSH and increases the affinity for glutamate (6).

The results of the present cross-sectional study indicated that GCLM mRNA was more abundant in tumor biopsies than in biopsies of adjacent tissues, whereas no significant differences in GCLC mRNA levels were observed between tumor and normal tissues. Although their expression is generally coordinated following stimulation, the two GCL subunits present distinct patterns of expression among different human tissues (5). This is partly due to the transcriptional control of these genes (5). Both GCLM and GCLC promoters contain the canonical antioxidant response element sequence, which is targeted by the transcription factor NRF2 (14). Upon oxidative stress, the NRF2 pathway is the major trigger of the antioxidant response (16). In addition, the two genes are also regulated by the NF- $\kappa \mathrm{B}$ pathway, which is another canonical salvage pathway against oxidative stress (15). NF- $\kappa \mathrm{B}$ signaling to GCL subunit promoters is mediated by the AP-1 pathway (10). However, the induction of this pathway was not evaluated in the present study, since the monitoring of the AP-1 pathway was not amenable to mRNA quantification (10). Both NRF2 and the $\mathrm{NF}_{-} \kappa \mathrm{B}$ are likely to be activated in $\mathrm{HN}$ cancer, since increased expression of NRF2 in $\mathrm{HN}$ tumors has been previously reported (30) and the dysregulation of the $N F-\kappa B$ pathway has been demonstrated to influence the progression of HN tumors (31). In the current study, no significant changes in the expression of the NRF2 and NF- $\mathrm{B}$ genes were detected, thus precluding any conclusion on the regulation of GCL by these pathways.

In addition, GCLM expression was restricted to basal cells in normal pluristratified epithelium, while it was broadly detected in dysplastic cells and non-differentiated tumor cells. The present observations are consistent with the pattern of GCL subunit expression in lung dysplasia, and confirmed earlier studies reporting expression of GCL in HN tumors $(32,33)$. Despite the mechanisms involved are unclear, the marked increase in GCLM expression in tumor biopsies may be responsible for the increased GSH levels in HN tumors, compared with normal tissues, observed in previous studies (18). Thus, GCLM modulation appears to be sufficient to produce significant changes in GSH synthesis (6). Under 
physiological conditions, GCL activity is the result of the GCLC/GCLM ratio, which mostly depends on the modulation of GCLM expression (34). In the present study, the expression of GCLM was heterogeneous within tumor lobules, whereby the periphery that was in close contact with the stroma exhibited the strongest labeling for GCLM. Notably, these regions were identified as the major sites of expression of Ki-67 (a well established cellular proliferation marker), in accordance with previous reports $(25,26,35)$. Therefore, the increased GCLM levels observed in the present study may be associated with the proliferative state of tumor cells, thus possibly linking cell proliferation with GSH levels (4). In the present study, the nuclear localization of GCLM is reported, which is in contradiction with the findings from previous studies conducted in Drosophila, where only GCLC was detected in the nucleus $(36,37)$. However, the pattern of expression of GCLC reported in that study hardly matched the distribution of GSH within mammalian dividing cells (38). Thus, although GSH is principally located in the nucleus of proliferating fibroblasts, GCLC is mainly located into the cytosol of Drosophila cells (36-38). Taken together, the importance of GCLM for GCL activity and the reported localization of GCLM may explain the high levels of GSH observed in the nucleus of proliferating cells. The presence of enzymes involved in the synthesis of GSH within the nucleus also explains the mechanism of GSH transport into the nucleus $(36,38)$.

In conclusion, the present study has demonstrated that the expression levels of GCLM within dysplastic and tumor cells derived from HN tumors are comparable with those observed in basal epithelial cells. The association of cell proliferation and GCL expression suggests that mechanisms involved in ensuring protection against oxidative stress are associated with HN tumor proliferation, which raises major concerns regarding individual variations in tumor cell resistance toward chemotherapy and radiotherapy among patients with HNSCC.

\section{Acknowledgements}

The present study was supported by the Scientific Research Fund of the Intermunicipal Public Health of Charleroi, University Hospital Center of Charleroi (Montigny-le-Tilleul, Belgium), the Fund for Medical Research in Hainaut (Mons, Belgium), the Fund for Cardiac Surgery (Brussels, Belgium) and the Institute of Pathology and Genetics (Gosselies, Belgium). L.V. is Research Director at the National Fund for Scientific Research (Brussels, Belgium).

\section{References}

1. Cooper JS, Porter K, Mallin K, Hoffman HT, Weber RS, Ang KK, Gay EG and Langer CJ: National Cancer Database report on cancer of the head and neck: 10-year update. Head Neck 31: 748-758, 2009.

2. Dandekar M and D'Cruz A: Organ preservation strategies: Review of literature and their applicability in developing nations. South Asian J Cancer 3: 147-150, 2014.

3. Gupta SC, Hevia D, Patchva S, Park B, Koh W and Aggarwal BB Upsides and downsides of reactive oxygen species for cancer: The roles of reactive oxygen species in tumorigenesis, prevention and therapy. Antioxid Redox Signal 16: 1295-1322, 2012.

4. Traverso N, Ricciarelli R, Nitti M, Marengo B, Furfaro AL, Pronzato MA, Marinari UM and Domenicotti C: Role of glutathione in cancer progression and chemoresistance. Oxid Med Cell Longev 2013: 972913, 2013.
5. Lu SC: Glutathione synthesis. Biochim Biophys Acta 1830: 3143-3153, 2013.

6. Chen Y, Shertzer HG, Schneider SN, Nebert DW and Dalton TP: Glutamate cysteine ligase catalysis: Dependence on ATP and modifier subunit for regulation of tissue glutathione levels. J Biol Chem 280: 33766-33774, 2005.

7. Dahl EL and Mulcahy RT: Cell-type specific differences in glutamate cysteine ligase transcriptional regulation demonstrate independent subunit control. Toxicol Sci 61: 265-272, 2001.

8. Cai J, Huang ZZ and Lu SC: Differential regulation of gamma-glutamylcysteine synthetase heavy and light subunit gene expression. Biochem J 326: 167-172, 1997.

9. Krzywanski DM, Dickinson DA, Iles KE, Wigley AF, Franklin CC, Liu RM, Kavanagh TJ and Forman HJ: Variable regulation of glutamate cysteine ligase subunit proteins affects glutathione biosynthesis in response to oxidative stress. Arch Biochem Biophys 423: 116-125, 2004.

10. Yang H, Magilnick N, Ou X and Lu SC: Tumour necrosis factor alpha induces co-ordinated activation of rat GSH synthetic enzymes via nuclear factor kappaB and activator protein-1. Biochem J 391: 399-408, 2005.

11. Wild AC, Moinova HR and Mulcahy RT: Regulation of gamma-glutamylcysteine synthetase subunit gene expression by the transcription factor Nrf2. J Biol Chem 274: 33627-33636, 1999.

12. Yang H, Wang J, Huang ZZ, Ou X and Lu SC: Cloning and characterization of the 5'-flanking region of the rat glutamate-cysteine ligase catalytic subunit. Biochem J 455: 447-455, 2001.

13. Reuter S, Gupta SC, Chaturvedi MM and Aggarwal BB: Oxidative stress, inflammation, and cancer: How are they linked? Free Radic Biol Med 49: 1603-1616, 2010.

14. Yang H, Magilnick N, Lee C, Kalmaz D, Ou X, Chan JY and Lu SC: Nrf1 and Nrf2 regulate rat glutamate-cysteine ligase catalytic subunit transcription indirectly via NF-kappaB and AP-1. Mol Cell Biol 25: 5933-5946, 2005.

15. Peng Z, Geh E, Chen L, Meng Q, Fan Y, Sartor M, Shertzer HG, Liu ZG, Puga A and Xia Y: Inhibitor of kappaB kinase beta regulates redox homeostasis by controlling the constitutive levels of glutathione. Mol Pharmacol 77: 784-792, 2010.

16. Baird L and Dinkova-Kostova AT: The cytoprotective role of the Keap1-Nrf2 pathway. Arch Toxicol 85: 241-272, 2011.

17. Na HK and Surh YJ: Oncogenic potential of Nrf2 and its principal target protein heme oxygenase-1. Free Radic Biol Med 67: 353-365, 2014.

18. Gamcsik MP, Kasibhatla MS, Teeter SD and Colvin OM: Glutathione levels in human tumors. Biomarkers 17: 671-691, 2012.

19. Dequanter D, Van de Velde M, Nuyens V, Nagy $N$, Van Antwerpen P, Vanhamme L, Zouaoui Boudjeltia K, Vanhaeverbeek M, Brohée D and Lothaire P: Assessment of oxidative stress in tumors and histologically normal mucosa from patients with head and neck squamous cell carcinoma: A preliminary study. Eur J Cancer Prev 22: 558-560, 2013.

20. van der Schroeff MP and Baatenburg de Jong RJ: Staging and prognosis in head and neck cancer. Oral Oncol 4-5: 356-360, 2009.

21. Livak KJ and Schmittgen TD: Analysis of relative gene expression data using real-time quantitative PCR and the 2(-Delta Delta C(T)) Method. Methods 25: 402-408, 2001.

22. Varghese F, Bukhari AB, Malhotra R and De A: IHC Profiler: An open source plugin for the quantitative evaluation and automated scoring of immunohistochemistry images of human tissue samples. PLoS One 9: e96801, 2014.

23. Sahoo P, Wilkins $\mathrm{C}$ and Yeager J: Threshold selection using Renyi's entropy. Pattern Recognit 30: 71-84, 1997.

24. Bottero V, Imbert V, Frelin C, Formento JL and Peyron JF: Monitoring NF-kappa B transactivation potential via real-time PCR quantification of I kappa B-alpha gene expression. Mol Diagn 7: 187-194, 2003.

25. Edström SS, Gustafsson B, Stenman G, Lydén E, Stein H and Westin T: Proliferative pattern of head and neck cancer. Am J Surg 162: 412-416, 1991.

26. Kearsley JH, Furlong KL, Cooke RA and Waters MJ: An immunohistochemical assessment of cellular proliferation markers in head and neck squamous cell cancers. Br J Cancer 61: 821-827, 1990.

27. Yellin SA, Davidson BJ, Pinto JT, Sacks PG, Qiao C and Schantz SP: Relationship of glutathione and glutathioneS-transferase to cisplatin sensitivity in human head and neck squamous carcinoma cell lines. Cancer Lett 85: 223-232, 1994. 
28. Kato T, Duffey DC, Ondrey FG, Dong G, Chen Z, Cook JA, Mitchell JB and Van Waes C: Cisplatin and radiation sensitivity in human head and neck squamous carcinomas are independently modulated by glutathione and transcription factor NF-kappaB. Head Neck 22: 748-759, 2000.

29. Bhattathiri VN, Sreelekha TT, Sebastian P, Remani P, Chandini R, Vijayakumar T and Nair MK: Influence of plasma GSH level on acute radiation mucositis of the oral cavity. Int J Radiat Oncol Biol Phys 29: 383-386, 1994

30. Stacy DR, Ely K, Massion PP, Yarbrough WG, Hallahan DE, Sekhar KR and Freeman ML: Increased expression of nuclear factor E2 p45-related factor 2 (NRF2) in head and neck squamous cell carcinomas. Head Neck 28: 813-818, 2006.

31. Loercher A, Lee TL, Ricker JL, Howard A, Geoghegen J, Chen Z, Sunwoo JB, Sitcheran R, Chuang EY, Mitchell JB, et al: Nuclear factor-kappaB is an important modulator of the altered gene expression profile and malignant phenotype in squamous cell carcinoma. Cancer Res 64: 6511-6523, 2004.

32. Kaarteenaho-Wiik R and Kinnula VL: Distribution of antioxidant enzymes in developing human lung, respiratory distress syndrome, and bronchopulmonary dysplasia. J Histochem Cytochem 52: 1231-1240, 2004.

33. Nishimura T, Newkirk K, Sessions RB, Andrews PA, Trock BJ, Rusmussen AA, Montogomery EA,BischoffEK, Hanigan MH and Cullen KJ: Association between expression of glutathione-associated enzymes and response to platinum-based chemotherapy in head and neck cancer. Chem Biol Interact 111-112: 187-198, 1998.
34. Lee JI, Kang J and Stipanuk MH: Differential regulation of glutamate-cysteine ligase subunit expression and increased holoenzyme formation in response to cysteine deprivation. Biochem J 393: 181-190, 2006.

35. Watanabe S, Watanabe R, Oton-Leite AF, Alencar RC, Oliveira JC, Leles CR, Batista AC and Mendonça EF: Analysis of cell proliferation and pattern of invasion in oral squamous cell carcinoma. J Oral Sci 52: 417-424, 2010.

36. Radyuk SN, Rebrin I, Luchak JM, Michalak K, Klichko VI, Sohal RS and Orr WC: The catalytic subunit of Drosophila glutamate-cysteine ligase is a nucleocytoplasmic shuttling protein. J Biol Chem 284: 2266-2274, 2009.

37. Markovic J, Borrás C, Ortega A, Sastre J, Viña J and Pallardó FV: Glutathione is recruited into the nucleus in early phases of cell proliferation. J Biol Chem 282: 20416-20424, 2007.

38. García-Giménez JL, Markovic J, Dasí F, Queval G, Schnaubelt D, Foyer $\mathrm{CH}$ and Pallardó FV: Nuclear glutathione. Biochim Biophys Acta 1830: 3304-3316, 2013. 